\title{
Crossed Transcortical Motor Aphasia, Left Spatial Neglect, and Limb and Magnetic Apraxia Due to Right Anterior Cerebral Artery Infarction
}

\author{
Hyoung Seop Kim, M.D., Jung Bin Shin, M.D., Ph.D., Jong Moon Kim, M.D.
}

Department of Rehabilitation Medicine, National Health Insurance Corporation Ilsan Hospital, Ilsan 410-719, Korea

\begin{abstract}
Crossed aphasia refers to language disturbance due to right-hemisphere lesions in right-handed individuals, while magnetic apraxia is described as 'forced grasping and groping' caused by lesions in the contralateral frontal lobe. This is a case report of a 70-year-old right handed woman who suffered from crossed transcortical motor aphasia and left hand magnetic apraxia due to right anterior cerebral artery infarction. The definite mechanism of this disorder is not yet understood, but neurophysiological observations suggest that affected supplementary motor areas may be responsible for this phenomenon.
\end{abstract}

Key Words Aphasia, Apraxia, Stroke

\section{INTRODUCTION}

The classic definition of crossed aphasia was an acquired language disturbance caused by a lesion in the cerebral hemisphere ipsilateral to the dominant hand. However, crossed aphasia is common in left handed people, and this term is now used exclusively to describe aphasia following a right hemisphere lesion in a right handed person. ${ }^{1,2}$

The definition of apraxia is the dysfunction of skilled

Received October 5, 2009; Accepted April 29, 2010

Corresponding author: Jong Moon Kim

Department of Rehabilitation Medicine, National Health Insurance Corporation Ilsan Hospital, 1232, Baeksok-dong, Ilsandong-gu, Goyang 410-719, Korea

Tel: +82-31-900-0670, Fax: +82-31-900-3343, E-mail: khan1013@hanmail. net

(c) This is an open-access article distributed under the terms of the Creative Commons Attribution Non-Commercial License (http:// creativecommons.org/licenses/by-nc/3.0) which permits unrestricted noncommercial use, distribution, and reproduction in any medium, provided the original work is properly cited.

Copyright $\odot 2011$ by Korean Academy of Rehabilitation Medicine movement not resulting from motor, sensory, or cognitive impairments after brain injury. ${ }^{3}$ Many different types of apraxia have been named and defined. Among them, magnetic apraxia was named and first used by Denny Brown to describe involuntarily forced grasping and groping movements of the hand against visual and tactile stimulation after medial frontal lobe injury. ${ }^{4}$ Aphasia and apraxia usually co-occur, although there is a reported case of exception. This phenomenon suggests that language and praxis share common mechanisms and common structures. ${ }^{5}$

We report one patient with right anterior cerebral artery infarction who demonstrated crossed transcortical motor aphasia, left limb apraxia, and magnetic apraxia. It is postulated that these abnormalities result from damage to the supplementary motor (SMA) area and cingulate cortex which are related to praxis and language function. 


\section{CASE REPORT}

A 70-year-old right-handed woman who suffered from Lt. hemiplegia due to Rt. ACA infarction was admitted to the in-patient department of rehabilitation medicine. According to her past medical history, she was identified as a chronic carrier of the hepatitis B virus 20 years ago and was diagnosed with essential hypertension, so she had been taking antihypertensive medicines. She was admitted to the university hospital on 24th, July 2008 due to acute onset of left leg weakness and dysarthria. At that time, her mental status was alert but she displayed apathy. Orientations to time and person were impaired, but orientation to place was intact. Calculation, repetition, and obedience to verbal commends were impaired, and urinary and fecal incontinence were intermittently noted. A cranial nerve function test didn't discover abnormal findings, and no definite extraocular movement limitation or facial palsy were noted. A manual muscle power test showed that the right upper and lower extremities were normal but the MRCs (Medical Research Council grade) of the left upper and lower extremities were Grade III and IV, respectively. The response to painful stimuli was slow in the left hemiside. Left side neglect such as tactile extinction was noted. Diffusion weighted MRI revealed acute infarction of the right medial frontal area, and she was admitted to the Department of Neurology and treated with intravenous heparin therapy. After 14 days, she was transferred to the Department of Rehabilitation Medicine (Fig. 1).

Psychological and speech evaluations were conducted. The Korean version of the Mini-Mental Status Examination (K-MMSE) score and the clinical dementia

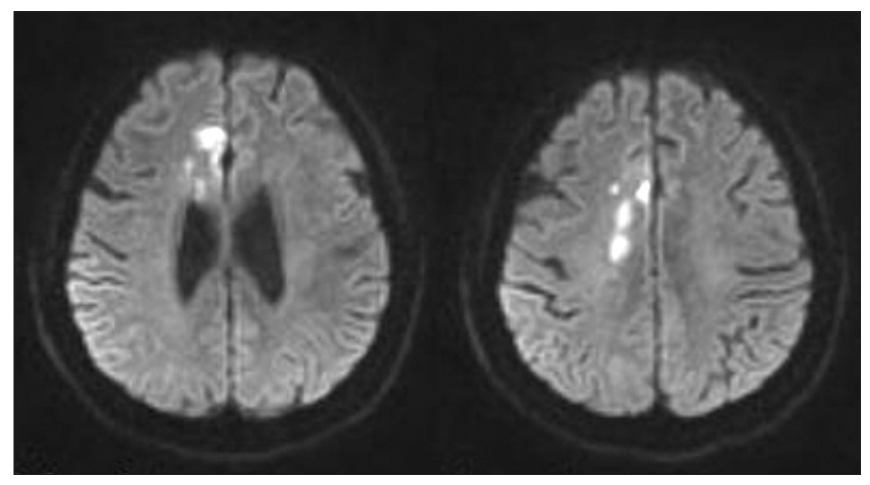

Fig. 1. Diffusion weighted brain MRI shows acute infarction in the vascular territory of right anterior cerebral artery. rating (CDR) were 8 and 2, respectively. Fist-edgepalm, alternating hand movement, alternating square \& triangle, and Luria loop tests were impaired, and preservation of verbal output was observed. In the speech evaluation, spontaneous speech, comprehension, and naming were poor, except for repetition. In a test for aspects of comprehension, the scores of yes or no used in replies to questions, perception of auditory words, and comprehension of orders were lower. She could obey a 1 step verbal commend such as "close your eyes," or "Raise your arm," but made mistakes responding to verbal commands that consisted of $>2$ steps and required comprehension of grammar and manipulation of objects. The aphasia quotient (AQ) in on the Korean version of the Western Aphasia Battery (K-WAB) was 25.8, and was indicative of severe to profound aphasia. The type of aphasia was considered to be crossed transcortical motor aphasia (Table 1).

All physical and neurological examinations were rechecked on 24 September, 2008. Follow-up scores of K-MMSE had improved to 15 , and she could voluntarily touch her head behind with her left upper extremity, however she was still resistant to passive movement such as "Gegenhalten". When the examiner touched her left palm with fingers or the handle of hammer, she grasped those as if "magnetically drawn," but she was unable to release the left grasping hand. She was able to transfer a pen from her right hand to her left hand, but it was

Table 1. The Results of Korean-Western Aphasia Battery on 3 Weeks and 7 Weeks after Onset

\begin{tabular}{lcc}
\hline \multirow{2}{*}{ Category of Paradise K-WAB } & \multicolumn{2}{c}{ After onset } \\
\cline { 2 - 3 } & 3 weeks & 7 weeks \\
\hline Spontaneous speech (20) & 2.5 & 10.0 \\
Information content (10) & 1.0 & 5.0 \\
\hline Fluency (10) & 1.5 & 5.0 \\
Comprehension (10) & 2.4 & 3.8 \\
Yes/no questions (3) & 2.0 & 2.1 \\
Auditory word recognition (3) & 0.3 & 1.3 \\
Sequential commands (4) & 0.1 & 0.4 \\
Repetition (10) & 6.6 & 8.7 \\
Naming (10) & 1.4 & 1.8 \\
Confrontation naming (6) & 1.4 & 1.6 \\
Generative naming (2) & 0 & 0 \\
\hline Sentence completion (1) & 0 & 0.2 \\
Responsive speech (1) & 0 & 0 \\
\hline
\end{tabular}


difficult when she did it inversely. The right hand would like to remove the pen from the left hand, but the left hand could not release it. Neglect was observed when she ate foods or did tasks during occupational therapy or psychological and speech evaluation, but the results of line bisection and the MVPT (motor visual perception test) were not obtained due to her poor cooperation. In the follow-up speech evaluation, all scores of subdivisions were improved, and the aphasia quotient (AQ) was 48.6.

\section{DISCUSSION}

The anterior cerebral artery supplies the rostral sensorimotor cortex and the anterior two-thirds of the corpus callosum by way of its calloso-marginal and pericallosal branches. Anterior cerebral artery lesions may destroy interhemispheric connections. ${ }^{6}$ If the lesions are located in the corpus callosum, the non-dominant hemisphere is disconnected from centers concerned with the organization of speech and skilled motor activity in the dominant hemisphere, resulting in agraphia and apraxia in the ipsilateral upper limb. ${ }^{6}$ The combination of these effects produces a unique and disabling disturbance of upper limb motor control, referred to as "Alien Hand Syndrome." In contrast to patients with callosal lesions or dominant medial frontal cortex lesions, the controlateral hand displays these abnormalities. These abnormal motor behaviors are considered to result from damage to the supplementary motor area. The main function of the SMA, which is connected with the primary motor cortex, is the planning and programming of coordinated motor tasks.

Lesions of the dominant SMA have been reported to cause mutism, transcortical motor aphasia, forced grasping, groping and a grasp reflex, reduced spontaneous motor activity, and impaired bimanual coordination. ${ }^{6}$ However, anterior cerebral artery occlusion may also produce transcortical motor aphasia with reduced, non-fluent spontaneous speech, but considerable preserved auditory comprehension. This type of aphasia has previously been ascribed to lesions involving the supplementary motor area or its connections with Broca's area. ${ }^{6}$

Crossed aphasia is rare, and its reported prevalence, has ranged from $0.4-2.0 \%{ }^{1}$ In Japan, a $1.1 \%$ prevalence rate for crossed aphasia was reported among 717 aphasia cases in a large Japanese rehabilitation hospital; ${ }^{1}$ however, in Korea, only 5 cases have been reported. ${ }^{2}$

Although the clinical phenomenology of crossed aphasia is established the "hereditary theory," also known as the "stockbrainedness theory," has been abandoned, because it stemmed from an incorrect belief that left-handers had language lateralized in the right hemisphere. However, Alexander and Annett suggested Annett's right shift theory to explain crossed aphasia. They believed that the theory could predict crossed aphasia by random lateralization in a small subset of the population in whom the right shift gene was absent. ${ }^{1,7,8}$ This gene confers some relative advantage on the left hemisphere which induces the left side to serve speech and gives a relative advantage to the right hand. It does not determine handedness, but it shifts a chance distribution of right handedness. ${ }^{7,8}$ If this gene is absent, lateralizations including language, handedness, and all other lateralized functions such as praxis, visuospatial function, and affective prosody function occur by chance; therefore all possible combinations of cerebral functional lateralizations may occur. ${ }^{8}$ The reason that they suggested their own theory was because previous "hereditary theory" has failed to provide a comprehensive explanation of the relationship between handedness and language in left- handers. Most left-handers who become aphasic also have left brain damage, but the incidence $(-30 \%)$ of aphasia after right brain damage is much higher in right-handers. Also, clinical phenomenology of aphasia in left-handers after right brain damage may not be identical to that after left brain damage. ${ }^{7,8}$ Crossed aphasia can be classified into 2 basic types; one is the "mirror image type" and the other is the "anomalous type". The former is similar to typical left brain damage aphasic profiles and the latter is similar to typical left brain damage profiles in addition to being crossed. However, any type of crossed aphasia has to be compatible with $50 \%$ occurrence of limb apraxia and right brain damage-type visuospatial deficits. ${ }^{7}$ Apraxia is common in clinical practice in association with aphasia, but is very seldom seen in isolation. ${ }^{5,9}$ Therefore, the frequent association between language dominance and handedness may also be an accident of anatomy, not a result of any fundamental interdependence between the two. ${ }^{9}$ Several cases of crossed aphasia support this view. These cases do not report any apraxic difficulties, consistent with the interpretation that the left hemisphere remained the repository of skilled motor functions despite language functions being localized to 
the right hemisphere. ${ }^{7,9}$

In our case, the patient showed combined symptoms of crossed aphasia, left side neglect, left limb ideamotor apraxia, and magnetic apraxia. Therefore, our case can be explained with the right shift theory because aphasia, limb and magnetic apraxia, and left sided visuospatial dysfunction were randomized.

She could give an imitation of using a comb and doing obeisance with her right hand, but she could not obey orders with her left hand. She intermittently raised her left upper extremity to touch her post head area (Fig. 2) and could toss a pen from her right hand to her left hand, but the opposite pattern was not observed. When she was asked to pass a pen from her left hand to her right hand, her left hand could not let it go (Fig. 3).

Lesions of the medial part of the frontal lobe may be responsible for magnetic apraxia. ${ }^{4,10}$ According to Lhermitte's paper, the affected areas of his 5 cases were the medial surface, the orbital surface, and the anterior pole of the frontal lobe. Because the lateral and medial cortex of the frontal lobes were normal, he suggested that it was probable that the cortex of the medial and lateral surfaces of the frontal lobes do not play a role in the liberation of the manual grasping behavior. In his cases, lesions of the anterior part of the corpus callosum were not observed. The lower frontal pole, the orbital surface of the frontal lobes, and the region of the Heubner's artery were necrotic. The role of the caudate nucleus is problematic, because it is connected by large fascicles to the orbital and medial areas. The pole and the orbital surface of the frontal lobe have a strong influence on motoricity. In our case, the lesion was localized in medial frontal area, but the corpus callosum was intact, so our case lends support to Lhemitte's opinion.

When treating ideamotor apraxia, it may helpful to provide general information about an activity's goal and omit specific instructions. Having the patient visualize task movements and sequences before carrying out the task may also be helpful. The visualization process gives the patient a visual model to refer to in performing the task. However, ideational apraxia involves a disruption in the concept formation of action planning. The ability to select and organize movements to execute an action is impaired. Treatment considerations for this dysfunction include step-by-step commands for each task, because patients with this disorder cannot grasp the general concept or idea of the activity, but may be able to perform individual components of the task on command. ${ }^{3}$

Our patient's score on an aphasia test battery, and her symptoms of left limb apraxia were improved. During
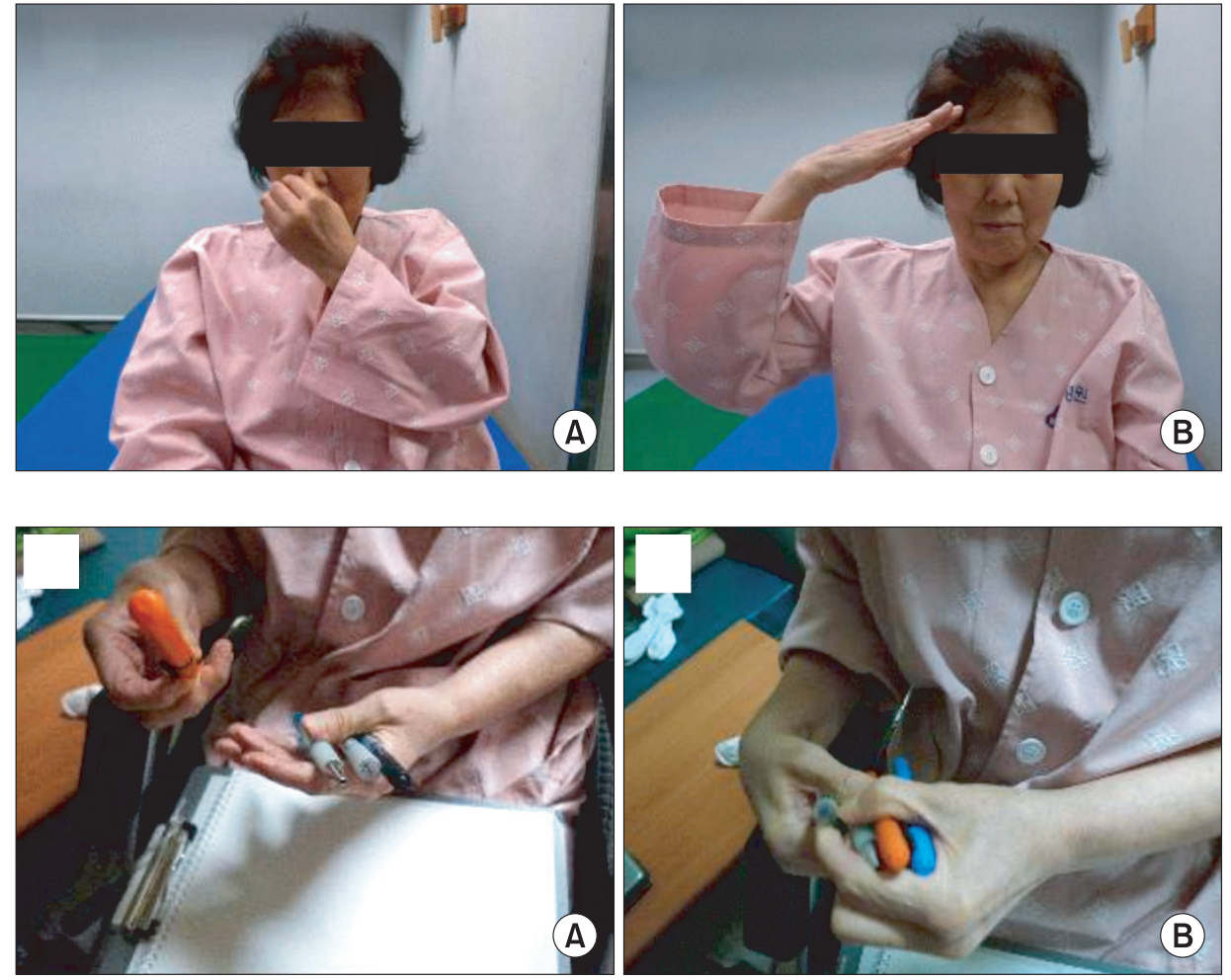

Fig. 2. (A) Saluting with the right hand. (B) Patient is able to touch her nose with her left hand on her will, but is not able to do so on command. Left spatial neglect and ideomotor apraxia were seen.

Fig. 3. (A) Transfering items from the right hand to the left is possible. (B) Difficulty on transfering items from the left hand to the right, due to the inability of the left hand to open up. Alien hand syndrome is seen. 

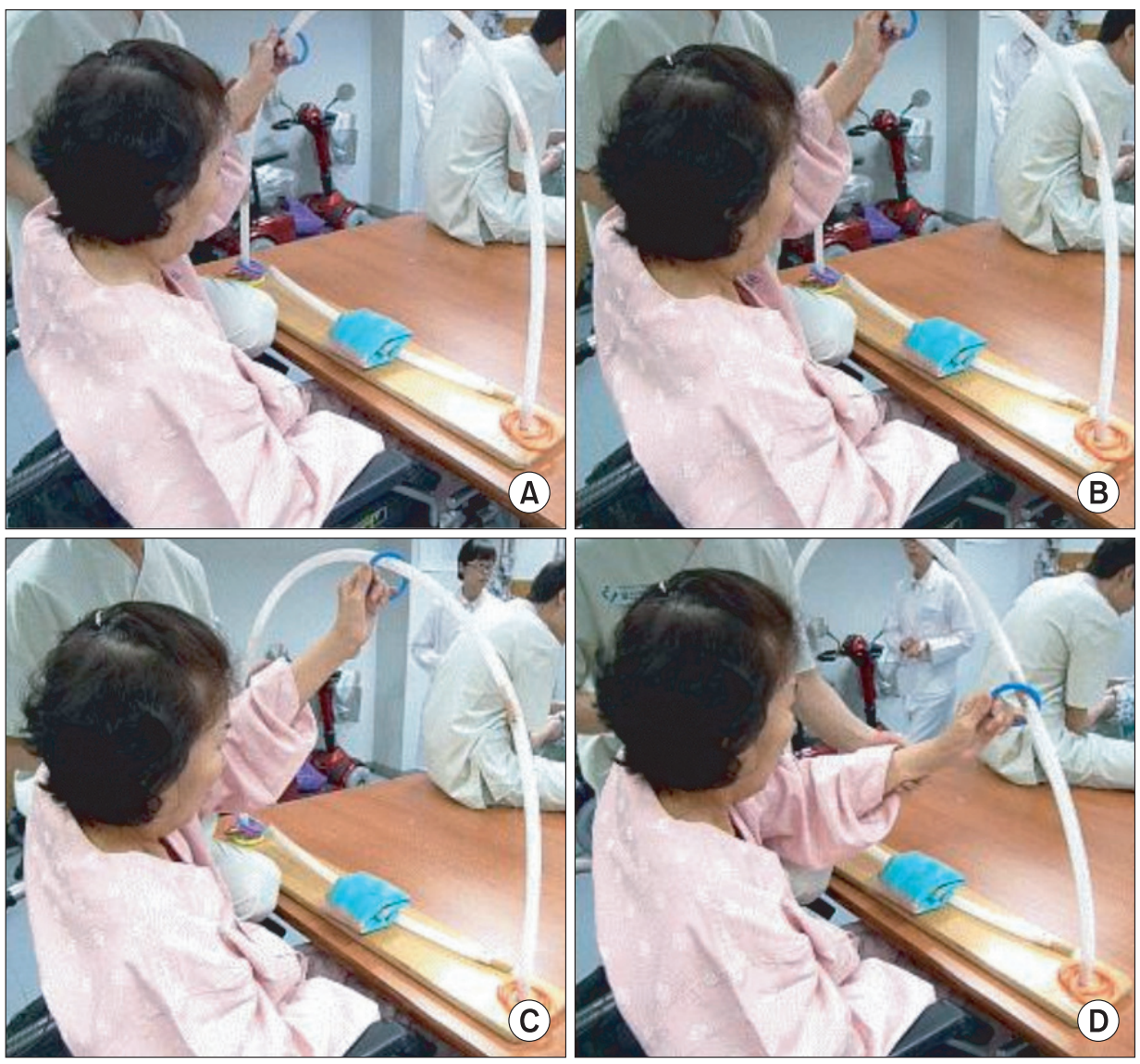

Fig. 4. From picture a to d in alphabetical order crossing the ring over the rim left to right side. Left spatial neglect is improved.

admission, she could move a ring with her left upper extremity, but magnetic apraxia had not disappeared (Fig. 4).

\section{REFERENCES}

1. Coppens P, Hungerford S, Yamaguchi S, Yamadori A. Crossed aphasia: an analysis of the symptoms, their frequency, and a comparison with left-hemisphere aphasia symptomatology. Brain Lang 2002; 83: 425463

2. Rho HJ, Kim YW, Park CI, Park JB, Jang JH. Nonfluent crossed aphasia after right middle cerebral artery infarction: a case report. J Korean Acad Rehab Med 2007; 31: 772-775

3. Gillen G, Burkhardt A. Stroke rehabilitation: a function-based approach, 1st ed, St Louis: Mosby, 1998, 161-184

4. Denny-Brown D. The nature of apraxia. J Nerv Ment Dis 1958; 126: 9-32
5. Papagno C, Della Sala S, Basso A. Ideomotor apraxia without aphasia and aphasia without apraxia: the anatomical support for a double dissociation. J Neurol Neurosurg Psychiatry 1993; 56: 286-289

6. McNabb AW, Carroll WM, Mastaglia FL. "Alien hand" and loss of bimanual coordination after dominant anterior cerebral artery territory infarction. J Neurol Neurosurg Psychiatry 1988; 51: 218-222

7. Annett M, Alexander MP. Atypical cerebral dominance: predictions and tests of the right shift theory. Neuropsychologia 1996; 34: 1215-1227

8. Alexander MP, Annett M. Crossed aphasia and related anomalies of cerebral organization: case reports and a genetic hypothesis. Brain Lang 1996; 55: 213-239

9. Selnes OA, Pestronk A, Hart J, Gordon B. Limb apraxia without aphasia from a left sided lesion in a right handed patient. J Neurol Neurosurg Psychiatry 1991; 54: 734-737

10. Lhermitte F. 'Utilization behaviour' and its relation to lesions of the frontal lobes. Brain 1983; 106: 237-255 